\title{
Estimativa do tamanho de parcela para experimentos com alface
}

\author{
Alessandro Dal'Col Lúcio; Fernando M Haesbaert; Daniel Santos; Vilson Benz \\ UFSM-CCR, Dep ${ }^{\text {to }}$ Fitotecnia, 97105-900 Santa Maria-RS; adlucio@ufsm.br (autor correspondente); fhaesbaert@gmail.com, \\ danielsantosagro@gmail.com, vibenz@gmail.com
}

\section{RESUMO}

O objetivo desse trabalho foi estimar o tamanho de parcela para a cultura da alface, cultivada em estufa, em túnel e a campo. Para tanto, foram conduzidos os seguintes experimentos com esta cultura: 1) em estufa no verão; 2) a campo no verão; 3 ) em estufa no inverno; 4) a campo no inverno; 5) em estufa no outono; 6) em túneis no outono; 7) em estufa na primavera e 8) em túneis na primavera. Foi avaliada a fitomassa fresca e seca da parte aérea das plantas. A partir das parcelas simuladas de diferentes tamanhos, foram estimados os parâmetros necessários para calcular o tamanho de parcela. Foi utilizado o método da máxima curvatura modificado para estimar o tamanho ótimo da parcela, e as diferenças mínimas significativas entre médias foram obtidas pelo método de Hatheway. Para testar a homogeneidade de variâncias entre as linhas de cultivo foi aplicado o teste de Bartlett. As parcelas devem estar dispostas no sentido da linha de cultivo. Os resultados mostraram que o tamanho ótimo de parcela para alface, independente de a variável ser a fitomassa fresca ou seca, ou da estação sazonal de cultivo, é de oito plantas para experimentos em estufa e em túneis e de nove plantas para experimentos a campo.

Palavras-chave: Lactuca sativa, ambiente protegido, planejamento, precisão experimental.

\section{ABSTRACT}

\section{Estimate of plot size for experiments with lettuce}

The aim of this study was to estimate the plot size for the lettuce crop grown in greenhouses, tunnel and field. The following experiments were carried out with this crop: 1) in greenhouse in summer; 2) in the field in summer; 3) in greenhouse in winter; 4) in the field in winter; 5) in greenhouse in autumn; 6) in tunnels in autumn; 7) in greenhouse in spring and; 8) in tunnels in spring. We evaluated the fresh and dry biomass of the shoots. We simulated plots of different sizes, from which we estimated the parameters necessary to estimate the plot size. We used the modified maximum curvature method to estimate the optimum plot size, and the least significant difference between means was obtained by the method of Hatheway. In order to test the homogeneity of variance between the rows of cultivation we applied the Bartlett test. The parcels shall be arranged in the direction of the crop row. In a simple recommendation, the optimum plot size for lettuce, regardless the variables fresh or dry weight as well as the season of cultivation, is of eight plants in the greenhouse and tunnel experiments, and nine plants for field experiments.

Keywords: Lactuca sativa, protected environment, planning, precision experiment.

\section{(Recebido para publicação em 17 de dezembro de 2010; aceito em 7 de outubro de 2011) (Received on December 17, 2010; accepted on October 7, 2011)}

A alface (Lactuca sativa) é apreciada em todo o mundo para o consumo em saladas frescas. Esta hortaliça geralmente é consumida in natura, sendo muito apreciada para o uso em dietas. No Brasil, é uma hortaliça de destaque, pois seu cultivo apresenta viabilidade econômica mesmo em pequenas propriedades e, além disso, devido à demanda de mão-de-obra relativamente alta, constitui uma importante fonte de empregos.

Nos meses de inverno, no sul do Brasil, existem períodos do ano com condições climáticas, que desfavorecem o desenvolvimento da cultura pela ocorrência de temperaturas inferiores a $10^{\circ} \mathrm{C}$ e precipitações pluviométricas prolongadas, ocorrendo danos físicos e retração do crescimento das plantas. Para a redução desses efeitos negativos sobre a cultura, uma das alternativas para viabilizar a produção nos meses desfavoráveis é o cultivo protegido, seja ele com túnel ou estufa, com o uso de filmes de polietileno de baixa densidade (PEBD).

Com a utilização do sistema de cultivo em ambientes protegidos, há a necessidade de aumentar a confiabilidade dos resultados obtidos em experimentos conduzidos nesses locais, ou seja, aumentar a qualidade dos experimentos, diminuindo o erro experimental. Uma das maneiras para que o erro experimental seja reduzido é a determinação do tamanho e da forma ótimos da parcela que, juntamente com o número de repetições, tem sido um dos principais problemas no planejamento de experimentos (Storck et al., 2006). Entretanto, muitas vezes os pesquisadores resolvem este problema empiricamente, usando tamanhos práticos no sentido da condução do experimento, da área disponível ou de sua experiência.
A variabilidade decresce com o aumento do tamanho da parcela; entretanto, com o aumento do tamanho da parcela, ocorre, paralelamente, grande aumento nos custos (Zhang et al., 1994). Devido a isso, é muito importante determinar o tamanho de parcela que possibilite a menor variabilidade com o mínimo de custos. O tamanho da parcela depende da heterogeneidade da área experimental (Lin et al., 1996), e essa pode se expressar de maneira distinta de uma região para outra ou com a mudança da cultura.

A determinação do tamanho e da forma das parcelas deve ser feita para cada cultura e para cada local em que ocorram condições climáticas e de solo diferentes das que já tenham sido determinadas (Oliveira \& Estefanel, 1995). Porém, como essas determinações vêm sendo estudadas principalmente nas grandes culturas (Resende \& Souza 
Júnior, 1997; Martin et al., 2005; Oliveira et al., 2006), para as olerícolas, especialmente aquelas cultivadas em ambiente controlado, existem poucas informações.

Vários trabalhos já foram realizados para determinar o tamanho de parcela para diversas culturas olerícolas (Lopes et al., 1998; Mello et al., 2004; Lorentz et al., 2005), que reforçam a idéia de que para cada cultura é necessário um tamanho de parcela específico, uma vez que o tamanho de parcela varia em função da cultura olerícola estudada. No entanto, não foram encontrados na literatura trabalhos indicando o tamanho de parcela para cultura da alface.

Diante da carência de técnicas experimentais específicas para cultura da alface cultivada em solo, principalmente quando realizados trabalhos em ambiente protegido, como estufas e túneis plásticos, o objetivo do trabalho foi estimar o tamanho ótimo de parcela para alface, cultivada em estufa, túnel e a campo.

\section{MATERIAL E MÉTODOS}

Os experimentos foram realizados na área experimental do Departamento de Fitotecnia no Campus da Universidade Federal de Santa Maria. Foram realizados os seguintes ensaios de uniformidade com alface: 1) em estufa no verão; 2) em estufa no inverno; 3) em estufa no outono; 4) em estufa na primavera; 5) a campo no verão; 6) a campo no inverno; 7) em túneis no outono; 8) em túneis na primavera. Estes experimentos foram realizados no ano de 2007 com a cultivar Vera, de 02/01 a 30/01/07 (verão) e de 16/06 a 19/07/07 (inverno) e, em 2008, com a cultivar Amanda, de 03/04 a 16/05/08 (outono) e de $10 / 10$ a 22/11/08 (primavera). As mudas utilizadas foram cultivadas em bandejas de 288 células com o uso do substrato HORTMIX. O transplante foi realizado quando as mudas estavam com 30 dias da emergência, apresentando três folhas.

A estufa plástica utilizada nos experimentos nas duas épocas foi a mesma que possuía estrutura metálica do tipo arco pampeano, pé direito de $2 \mathrm{~m}$ e 3,5 m na parte central, com 20 m de comprimento e orientada no sentido norte-sul. Os túneis plásticos utilizados nos experimentos também eram idênticos: ambos possuíam pé direito de $3 \mathrm{~m}, 20 \mathrm{~m}$ de comprimento e orientação norte-sul. A cobertura destes ambientes protegidos foi realizada com filme de polietileno de baixa densidade (PEBD), com espessura de 100 micras e aditivo anti-UV.

Em todos os experimentos, as plantas foram dispostas em linhas espaçadas de um metro, com espaçamento entre plantas de $0,3 \mathrm{~m}$. Nos experimentos realizados em estufa plástica foram utilizadas seis linhas de cultivo cada uma contendo 48 plantas. Nos realizados em túneis, foram usadas três linhas de cultivo cada uma com 60 plantas. Nos realizados a campo, no verão foram utilizadas seis linhas e no inverno três linhas, cada uma contendo 48 plantas. As irrigações foram feitas através de gotejamento e os demais tratos culturais foram realizados de acordo com as recomendações para a cultura (Filgueira, 2000).

Para determinar as fitomassas fresca (FF) e seca (FS) da parte aérea, foram colhidas as partes aéreas de todas as plantas de cada experimento no período da manhã. Estas plantas foram acondicionadas em sacos de papel e pesadas em balança digital com precisão de 1 g para determinação da FF. Após a primeira pesagem, as partes aéreas foram levadas para estufa de circulação de ar forçado à temperatura de $65 \pm 0,5^{\circ} \mathrm{C}$, por 72 horas, quando atingiram massa constante para, em seguida, proceder a segunda pesagem para determinação da FS por planta.

Os diferentes tamanhos de parcela $(\mathrm{X})$, múltiplos do número de unidades básicas (UB) por linha de cultivo, foram constituídos pela soma da produção obtida nas UB vizinhas na linha de cultivo. Foram estabelecidos $\mathrm{X}=1,2,3,4,6,8 \mathrm{e}$ 12 UB para os experimentos em estufa e a campo e $\mathrm{X}=1,2,3,4,5,6$ e $10 \mathrm{UB}$ nos experimentos em túneis. Para cada valor de X foram calculados: $\mathrm{M}(\mathrm{x})$, média das parcelas com X UB de tamanho; V(x), variância entre as parcelas de X UB de tamanho; $\mathrm{CV}(\mathrm{x})$, coeficiente de variação entre as parcelas de X UB de tamanho; e $\mathrm{VU}(\mathrm{x})=\mathrm{V}(\mathrm{x}) / \mathrm{X}^{2}$, variância por $\mathrm{UB}$ entre as parcelas de X UB de tamanho.

O valor do índice de heterogeneidade do solo (b) foi estimado via logaritimização da função $\mathrm{VU}(\mathrm{x})=V 1 / X^{b}($ Smith, 1938), ou seja, $\log (\mathrm{VU}(\mathrm{x}))=\log (\mathrm{V} 1)-\mathrm{b} \log (\mathrm{X})$, cuja estimação dos parâmetros (V1 e b) foi ponderada pelos graus de liberdade, associados a cada um dos tamanhos de parcela (Steel et al., 1997). De forma semelhante, estimaram-se os parâmetros da função $\mathrm{CV}(\mathrm{x})=A / X^{B}$ (Lessman \& Atkins, 1963).

O tamanho ótimo de parcela foi estimado pelo método da máxima curvatura modificado por Meier \& Lessman (1971), pela equação $\mathrm{X}_{0}=\left\{\mathrm{A}^{2} \mathrm{~B}^{2}(2 \mathrm{~B}+1) /(\mathrm{B}+2)\right\}^{1 /(2 \mathrm{~B}+2)}$. $\mathrm{A}$ diferença mínima significativa entre médias, em percentagem da média (D), foi calculada pelo método de Hatheway (1961) $\mathrm{D}=2(\mathrm{t} 1+\mathrm{t} 2)^{2} \mathrm{~A}^{2} / \mathrm{rX}^{\mathrm{b}}$, em que t1 e t2 são valores tabelados da distribuição t-Student com nível de erro 0,05 e 0,4 , respectivamente; $r$ é o número de repetições, tomado como igual ao número de linhas de cultivo do experimento; X é o tamanho da parcela. Os valores tabelados de distribuição de $\mathrm{t}$ foram obtidos através dos graus de liberdade obtidos pela expressão $\mathrm{g} 1=\mathrm{I}(\mathrm{r}-1)$, onde I é o número de tratamentos e r é o número de repetições.

Para testar a homogeneidade de variâncias entre as linhas de cultivo foram realizados testes de Bartlett (Steel et al., 1997) a 5\% de probabilidade de erro, que é um teste adequado para testar mais de duas variâncias ao mesmo tempo.

\section{RESULTADOS E DISCUSSÃO}

Os resultados dos testes de Bartlett realizados dentro de cada experimento, entre as variâncias das linhas de cultivo com parcelas de uma UB, mostraram que nos cultivos no ano de 2007 , considerando as duas variáveis mensuradas, as variâncias foram heterogêneas em $50 \%$ dos experimentos no verão e em $75 \%$ no inverno. Nos ensaios de 2008 , a heterogeneidade foi verificada em $33 \%$ no outono e em $50 \%$ na primavera (Tabelas 1 e 2). Esses resultados evidenciam que a estação sazonal de cultivo influenciou as variabilidades entre as 
Tabela 1. Número de unidades básicas por parcela $(X)$, média $(\bar{X})$, variância por unidade básica $(V U(x))$ e coeficiente de variação $(C V$ \%) por parcela simulada e índice de heterogeneidade (b) para as fitomassas fresca (FF) e seca (FS) da parte aérea de alface, produzidas em estufa e a campo, em duas estações sazonais do ano de 2007 (number of basic units per plot $(\mathrm{X})$, average value $(\overline{\mathrm{X}})$, variance per basic unit (VU (x)) and coefficient of variation (CV \%) per plot simulated and index of soil heterogeneity (b) for the fresh (FF) and dry mass (FS) from the shoots of lettuce, grown in greenhouse and in the field in two seasons of the year 2007). Santa Maria, UFSM, 2010.

\begin{tabular}{|c|c|c|c|c|c|c|c|c|c|c|c|c|}
\hline \multicolumn{13}{|c|}{ Estufa } \\
\hline \multirow[b]{3}{*}{$\mathbf{X}$} & \multicolumn{6}{|c|}{ Verão } & \multicolumn{6}{|c|}{ Inverno } \\
\hline & \multicolumn{3}{|c|}{ FF } & \multicolumn{3}{|c|}{ FS $^{*}$} & \multicolumn{3}{|c|}{ FF } & \multicolumn{3}{|c|}{ FS* } \\
\hline & $\overline{\bar{X}}$ & $V(x)$ & $\mathrm{CV}$ & $\overline{\bar{X}}$ & $V(x)$ & $\mathrm{CV}$ & $\overline{\bar{X}}$ & $\mathbf{V}(\mathbf{x})$ & $\mathrm{CV}$ & $\overline{\bar{X}}$ & $\mathrm{~V}(\mathbf{x})$ & $\mathrm{CV}$ \\
\hline 1 & 236,3 & 7131,7 & 36 & 14,3 & 18,7 & 30 & 123,0 & 1802,0 & 35 & 10,7 & 5,0 & 21 \\
\hline 2 & 475,8 & 5343,3 & 31 & 28,8 & 17,0 & 29 & 245,9 & 910,9 & 25 & 21,4 & 4,5 & 20 \\
\hline 3 & 708,8 & 4136,9 & 27 & 42,9 & 13,3 & 25 & 368,9 & 534,1 & 19 & 32,0 & 3,9 & 19 \\
\hline 4 & 945,1 & 4647,3 & 29 & 57,2 & 17,6 & 29 & 491,8 & 502,0 & 18 & 42,7 & 4,3 & 19 \\
\hline 6 & 1417,6 & 2560,9 & 21 & 85,8 & 8,1 & 20 & 737,8 & 333,9 & 15 & 64,1 & 3,3 & 17 \\
\hline 8 & 1830,6 & 1925,8 & 19 & 112,9 & 8,2 & 20 & 955,3 & 314,7 & 15 & 84,2 & 2,7 & 15 \\
\hline 12 & 2835,2 & 956,1 & 13 & 171,6 & 5,8 & 17 & 1475,5 & 235,6 & 12 & 128,1 & 2,7 & 15 \\
\hline $\mathrm{b}$ & \multicolumn{3}{|c|}{1,317} & \multicolumn{3}{|c|}{0,552} & \multicolumn{3}{|c|}{1,468} & \multicolumn{3}{|c|}{0,336} \\
\hline \multicolumn{13}{|c|}{ Campo } \\
\hline & \multicolumn{6}{|c|}{ Verão } & \multicolumn{6}{|c|}{ Inverno } \\
\hline & \multicolumn{3}{|c|}{ FF } & \multicolumn{3}{|c|}{ FS* } & \multicolumn{3}{|c|}{ FF* } & \multicolumn{3}{|c|}{ FS* } \\
\hline $\mathbf{X}$ & $\overline{\bar{X}}$ & $V(x)$ & $\mathrm{CV}$ & $\overline{\bar{X}}$ & $V(x)$ & $\mathrm{CV}$ & $\overline{\bar{X}}$ & $V(x)$ & $\mathrm{CV}$ & $\overline{\bar{X}}$ & $\mathrm{~V}(\mathbf{x})$ & $\mathrm{CV}$ \\
\hline 1 & 110,5 & 3337,2 & 52 & 7,7 & 11,0 & 43 & 57,4 & 667,3 & 45 & 6,7 & 4,1 & 30 \\
\hline 2 & 230,5 & 2080,0 & 40 & 16,1 & 8,2 & 36 & 114,8 & 500,7 & 39 & 13,4 & 3,8 & 29 \\
\hline 3 & 334,9 & 1598,5 & 36 & 23,3 & 7,4 & 35 & 172,2 & 389,3 & 34 & 20,1 & 3,3 & 27 \\
\hline 4 & 441,8 & 2050,0 & 41 & 30,8 & 10,2 & 41 & 229,6 & 371,5 & 34 & 26,8 & 3,7 & 29 \\
\hline 6 & 662,8 & 1166,2 & 31 & 46,2 & 5,2 & 30 & 344,4 & 325,8 & 31 & 40,3 & 2,7 & 24 \\
\hline 8 & 873,3 & 1048,9 & 30 & 61,0 & 5,2 & 30 & 449,7 & 261,5 & 29 & 52,7 & 2,0 & 21 \\
\hline 12 & 1325,5 & 545,4 & 21 & 92,4 & 2,8 & 22 & 688,8 & 218,1 & 26 & 80,5 & 1,3 & 17 \\
\hline $\mathrm{b}$ & & 1,244 & & & 0,478 & & & 0,990 & & & 0,323 & \\
\hline
\end{tabular}

*variâncias heterogêneas entre linhas de cultivo pelo teste de Bartlett a 5\% de probabilidade de erro (heterogeneous variances between lines of cultivation by the Bartlett test at $5 \%$ probability of error).

linhas de cultivo, concordando com os trabalhos de Lúcio et al. (2008), Carpes et al. (2008) e Carpes et al. (2010) com abobrinha italiana, e Santos et al. (2010) com alface. Como não foi constatada a homogeneidade das variâncias entre linhas de cultivo na totalidade dos testes, as parcelas neste tipo de experimento deverão estar dispostas no sentido da linha de cultivo.

Pelos resultados das Tabelas 1 e 2 verifica-se que, de maneira geral, o aumento no número de unidades básicas por parcela, levou a uma redução na variância por unidade básica entre as parcelas (VU(x)). Os valores de coeficiente de variação $(\mathrm{CV})$ também diminuíram concordando com os trabalhos de Oliveira \& Estefanel (1995), Zanon \& Storck (2000) e Lúcio et al. (2004), que trabalharam com batata, eucalipto e pimentão, respectivamente.

$\mathrm{Na}$ maioria dos experimentos, os CV obtidos para a variável fitomassa fresca (FF), foram maiores do que os obtidos para a fitomassa seca (FS) (Tabelas 1 e 2). Isto deve ter ocorrido porque a alface possui em média 94\% de água em sua constituição (Ohse et al., 2001), e por isso, parte da variabilidade inerente a esta cultura ocorre devido a variações do teor de água de uma planta para outra. Dessa forma, quando a água é extraída, a variabilidade é diminuída. Em alguns casos, os $\mathrm{CV}$ para a FF foram menores que para a $\mathrm{FS}$, e isso ocorreu porque nesses casos a redução da variabilidade proporcionada pela utilização FS não foi suficiente para compensar as médias mais elevadas, que ocorrem quando é usado a FF.

Nos experimentos realizados em 2007, os valores do índice de heterogeneidade do solo (b) foram maiores no experimento em estufa, em que o b foi em média de 1,4, enquanto que no campo foi em média de 1,2 (Tabela 1). Lopes et al. (1998) e Lúcio et al. (2004) obtiveram b superiores a 0,6 em seus estudos em estufa plástica, com tomate salada e pimentão, respectivamente. Estes resultados mostram que a área experimental delimitada por uma estufa plástica não gera uma área experimental homogênea. Em todos os experimentos, os valores de $b$ variaram em função da variável resposta, sendo que nos experimentos de 2007 os b variaram mais de $50 \%$ com a alteração da variável. Também houve variação dos b entre 
Tabela 2. Número de unidades básicas por parcela $(X)$, média $(\bar{X})$, variância por unidade básica $(V U(x))$ e coeficiente de variação $(C V$ \%) por parcela simulada e índice de heterogeneidade (b) para as fitomassas fresca (FF) e seca (FS) da parte aérea de alface, produzidas em estufa e em túneis, em duas estações sazonais do ano de 2008 (number of basic units per plot (X), average value ( $\overline{\mathrm{X}})$, variance per basic unit (VU (x)) and coefficient of variation (CV\%) per plot simulated and index of soil heterogeneity (b) for the fresh (FF) and dry mass (FS) from the shoots of lettuce, grown in greenhouses and tunnels, two seasons of the year 2008). Santa Maria, UFSM, 2010.

\begin{tabular}{|c|c|c|c|c|c|c|c|c|c|c|c|c|}
\hline \multicolumn{13}{|c|}{ Estufa } \\
\hline \multirow[b]{3}{*}{$\mathbf{X}$} & \multicolumn{6}{|c|}{ Outono } & \multicolumn{6}{|c|}{ Primavera } \\
\hline & \multicolumn{3}{|c|}{ FF* $^{*}$} & \multicolumn{3}{|c|}{ FS } & \multicolumn{3}{|c|}{ FF* $^{*}$} & \multicolumn{3}{|c|}{ FS* } \\
\hline & $\bar{X}$ & $V(x)$ & $\mathrm{CV}$ & $\bar{X}$ & $V(x)$ & $\mathrm{CV}$ & $\bar{X}$ & $V(x)$ & $\mathrm{CV}$ & $\bar{X}$ & $V(x)$ & $\mathrm{CV}$ \\
\hline 1 & 249,9 & 11119,1 & 42 & 11,0 & 17,1 & 37 & 405,3 & 19769,1 & 35 & 21,3 & 44,4 & 31 \\
\hline 2 & 499,8 & 7608,5 & 35 & 22,1 & 10,2 & 29 & 810,6 & 14078,5 & 29 & 42,7 & 25,6 & 24 \\
\hline 3 & 749,7 & 6641,4 & 33 & 33,1 & 7,7 & 25 & 1215,9 & 11945,9 & 27 & 64,0 & 19,9 & 21 \\
\hline 4 & 999,6 & 6260,3 & 32 & 44,1 & 7,3 & 24 & 1621,3 & 11502,3 & 26 & 85,3 & 19,1 & 20 \\
\hline 6 & 1499,5 & 5506,6 & 30 & 66,2 & 5,9 & 22 & 2431,9 & 10224,3 & 25 & 128,0 & 15,3 & 18 \\
\hline 8 & 1954,0 & 5031,3 & 29 & 86,8 & 5,9 & 22 & 3168,0 & 8649,9 & 23 & 166,0 & 11,1 & 16 \\
\hline 12 & 2998,9 & 4901,4 & 28 & 132,4 & 5,2 & 21 & 4863,8 & 8547,7 & 23 & 255,9 & 11,5 & 16 \\
\hline $\mathrm{b}$ & \multicolumn{3}{|c|}{1,272} & \multicolumn{3}{|c|}{0,728} & \multicolumn{3}{|c|}{1,327} & \multicolumn{3}{|c|}{0,869} \\
\hline \multicolumn{13}{|c|}{ Túnel 1} \\
\hline & \multicolumn{6}{|c|}{ Outono } & \multicolumn{6}{|c|}{ Primavera } \\
\hline & \multicolumn{3}{|c|}{ FF* } & \multicolumn{3}{|c|}{ FS } & \multicolumn{3}{|c|}{ FF } & \multicolumn{3}{|c|}{ FS* } \\
\hline $\mathbf{X}$ & $\bar{X}$ & $V(x)$ & $\mathrm{CV}$ & $\bar{X}$ & $V(x)$ & $\mathrm{CV}$ & $\overline{\mathrm{X}}$ & $V(x)$ & $\mathrm{CV}$ & $\overline{\mathrm{X}}$ & $V(x)$ & $\mathrm{CV}$ \\
\hline 1 & 208,0 & 10491,2 & 49 & 10,3 & 10,8 & 32 & 536,3 & 49798,3 & 42 & 30,7 & 107,6 & 34 \\
\hline 2 & 415,9 & 5375,3 & 35 & 20,6 & 6,5 & 25 & 1072,5 & 39614,9 & 37 & 61,4 & 77,9 & 29 \\
\hline 3 & 623,9 & 4132,9 & 31 & 30,9 & 4,7 & 21 & 1608,8 & 36346,0 & 36 & 92,1 & 66,6 & 27 \\
\hline 4 & 831,8 & 3221,1 & 27 & 41,1 & 3,8 & 19 & 2145,0 & 32490,8 & 34 & 122,8 & 59,9 & 25 \\
\hline 5 & 1039,8 & 2108,9 & 22 & 51,4 & 2,4 & 15 & 2681,3 & 31838,7 & 33 & 153,5 & 56,1 & 24 \\
\hline 6 & 1247,8 & 2008,8 & 22 & 61,7 & 2,9 & 17 & 3217,5 & 32379,4 & 34 & 184,2 & 57,4 & 25 \\
\hline 10 & 2079,6 & 1018,4 & 15 & 102,8 & 1,9 & 13 & 5362,5 & 28815,4 & 32 & 307,0 & 50,4 & 23 \\
\hline $\mathrm{b}$ & & 916 & & & 798 & & & 275 & & & 397 & \\
\hline
\end{tabular}

Túnel 2

\begin{tabular}{|c|c|c|c|c|c|c|c|c|c|c|c|c|}
\hline \multirow[b]{3}{*}{$\mathbf{X}$} & \multicolumn{6}{|c|}{ Outono } & \multicolumn{6}{|c|}{ Primavera } \\
\hline & \multicolumn{3}{|c|}{ FF } & \multicolumn{3}{|c|}{ FS } & \multicolumn{3}{|c|}{ FF } & \multicolumn{3}{|c|}{ FS } \\
\hline & $\overline{\mathrm{X}}$ & $V(x)$ & $\mathrm{CV}$ & $\bar{X}$ & $V(x)$ & $\mathrm{CV}$ & $\overline{\mathrm{X}}$ & $V(x)$ & $\mathrm{CV}$ & $\overline{\mathrm{X}}$ & $V(x)$ & $\mathrm{CV}$ \\
\hline 1 & 302,0 & 13628,8 & 39 & 15,3 & 32,7 & 37 & 686,2 & 28317,7 & 25 & 37,3 & 68,0 & 22 \\
\hline 2 & 603,9 & 8930,5 & 31 & 30,6 & 19,5 & 29 & 1372,3 & 16609,1 & 19 & 74,7 & 37,4 & 16 \\
\hline 3 & 905,9 & 7662,7 & 29 & 46,0 & 16,1 & 26 & 2058,5 & 9650,6 & 14 & 112,0 & 24,1 & 13 \\
\hline 4 & 1207,8 & 6567,2 & 27 & 61,3 & 13,4 & 24 & 2744,6 & 8444,2 & 13 & 149,4 & 20,8 & 12 \\
\hline 5 & 1509,8 & 5066,5 & 24 & 76,6 & 10,0 & 21 & 3430,8 & 7442,8 & 13 & 186,7 & 15,6 & 11 \\
\hline 6 & 1811,7 & 5278,5 & 24 & 91,9 & 10,1 & 21 & 4117,0 & 7137,7 & 12 & 224,0 & 15,5 & 11 \\
\hline 10 & 3019,6 & 3935,8 & 21 & 153,2 & 6,7 & 17 & 6861,6 & 5989,5 & 11 & 373,4 & 12,5 & 9 \\
\hline b & \multicolumn{3}{|c|}{0,553} & \multicolumn{3}{|c|}{0,675} & \multicolumn{3}{|c|}{0,843} & \multicolumn{3}{|c|}{0,876} \\
\hline
\end{tabular}

*variâncias heterogêneas entre linhas de cultivo pelo teste de Bartlett a 5 \% de probabilidade de erro. (heterogeneous variances between lines of cultivation by the Bartlett test at $5 \%$ probability of error).

as estações de cultivo. Estes resultados evidenciam que para cada variável, ambiente ou estação de cultivo, é necessário uma estimativa distinta de b. Este é um dos motivos para as diferenças que ocorreram nos tamanhos ótimos de par- cela e nos D (diferença em porcentagem da média) entre variáveis, ambientes e estações de cultivo (Tabela 3).

Segundo Lin \& Binns (1986), quando $\mathrm{b}$ for maior que 0,7 , o aumento do tamanho da parcela é mais eficiente na melhoria da precisão em uma área experimental. Quando b for menor que 0,2 , melhores resultados serão obtidos com o aumento do número de repetições e, para valores intermediários de $\mathrm{b}$, ambas as estratégias serão eficientes. Do total de 
Tabela 3. Diferença mínima significativa entre médias (D \%), para cada tamanho de parcela (X), e tamanho ótimo da parcela (Xo) em número de unidades básicas, para as fitomassas fresca (FF) e seca (FS) de alface cultivada em diferentes ambientes em quatro estações sazonais e em dois anos (least significant difference between means (D \%) for each plot size (X), and plot size (Xo) in number of basic units, for the fresh (FF) and dry mass (FS) of lettuce grown in different environments in four seasons and in two years). Santa Maria, UFSM, 2010.

\begin{tabular}{|c|c|c|c|c|c|c|c|c|c|c|c|c|c|}
\hline \multicolumn{14}{|c|}{2007} \\
\hline \multirow[b]{3}{*}{$\mathbf{X}$} & \multicolumn{7}{|c|}{ Verão } & \multicolumn{6}{|c|}{ Inverno } \\
\hline & \multicolumn{4}{|c|}{ Estufa } & \multicolumn{3}{|c|}{ Campo } & \multicolumn{3}{|c|}{ Estufa } & \multicolumn{3}{|c|}{ Campo } \\
\hline & & FF & \multicolumn{2}{|c|}{ FS } & FF & \multicolumn{2}{|c|}{ FS } & \multicolumn{2}{|c|}{ FF } & FS & \multicolumn{2}{|r|}{ FF } & \multirow[t]{2}{*}{ FS } \\
\hline \multirow[b]{2}{*}{1} & \multicolumn{12}{|c|}{ D } & \\
\hline & & 67,0 & \multicolumn{2}{|c|}{57,3} & 96,1 & \multicolumn{2}{|c|}{80,1} & \multicolumn{2}{|c|}{61,4} & 38,7 & \multicolumn{2}{|r|}{119,5} & 81,4 \\
\hline 2 & & 42,6 & \multicolumn{2}{|c|}{47,5} & 62,7 & \multicolumn{2}{|c|}{68,1} & \multicolumn{2}{|c|}{37,0} & 34,6 & \multicolumn{2}{|r|}{85,7} & 73,6 \\
\hline 3 & & 32,8 & \multicolumn{2}{|c|}{42,7} & 48,9 & \multicolumn{2}{|c|}{62,1} & \multicolumn{2}{|c|}{27,6} & 32,5 & \multicolumn{2}{|r|}{70,9} & 69,6 \\
\hline 4 & & 27,2 & &, 6 & 41,1 & & & 22 & & 31,1 & & 62,1 & 67,2 \\
\hline 6 & & 21,0 & 3. &, 7 & 32,2 & & & 16 & & 29,3 & & 51,9 & 64,3 \\
\hline 8 & & 17,5 & 3. & 2 & 27,1 & & & 13 & & 28,1 & & 46,0 & 62,7 \\
\hline 12 & & 13,7 & &, 2 & 21,5 & & & 10 & & 26,7 & & 39,4 & 61,5 \\
\hline $\mathrm{X}_{0}$ & & 7 & 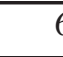 & & 9 & & & 7 & & 5 & & 8 & 6 \\
\hline & & & & & & & 2008 & & & & & & \\
\hline & & & & Out & & & & & & Pri & avera & & \\
\hline & & ufa & & Túr & & & el 2 & & ufa & & & & el 2 \\
\hline $\mathbf{X}$ & FF & FS & $\mathbf{X}$ & FF & FS & FF & FS & FF & FS & FF & FS & FF & FS \\
\hline & & & & & & & & & & & & & \\
\hline 1 & 77,9 & 67,7 & 1 & 113,7 & 74,1 & 88,9 & 85,8 & 63,8 & 56,2 & 95,5 & 77,3 & 56,5 & 50,8 \\
\hline 2 & 50,3 & 52,8 & 2 & 83,4 & 56,7 & 74,0 & 68,5 & 40,4 & 41,7 & 87,5 & 68,0 & 42,5 & 37,8 \\
\hline 3 & 39,0 & 45,8 & 3 & 69,9 & 48,6 & 66,7 & 60,2 & 31,0 & 35,1 & 83,5 & 63,2 & 36,1 & 31,9 \\
\hline 4 & 32,6 & 41,4 & 4 & 61,7 & 43,7 & 62,1 & 55,1 & 25,7 & 31,1 & 80,9 & 60,2 & 32,3 & 28,4 \\
\hline 6 & 25,4 & 36,0 & 5 & 56,2 & 40,3 & 58,9 & 51,6 & 19,8 & 26,3 & 79,1 & 58,1 & 29,6 & 26,0 \\
\hline 8 & 21,4 & 32,7 & 6 & 52,2 & 37,8 & 56,5 & 48,9 & 16,5 & 23,4 & 77,8 & 56,5 & 27,7 & 24,2 \\
\hline 12 & 16,8 & 28,7 & 10 & 42,8 & 31,9 & 50,8 & 42,6 & 12,9 & 20,0 & 75,2 & 52,9 & 23,1 & 20,0 \\
\hline $\mathrm{X}_{0}$ & 8 & 7 & & 8 & 6 & 5 & 6 & 7 & 6 & 4 & 4 & 5 & 4 \\
\hline
\end{tabular}

b estimados, $60 \%$ apresentaram valores superiores a 0,7 e o restante apresentou valores entre 0,2 e 0,7 (Tabelas 1 e $2)$. Esses resultados indicam que, em experimentos com a cultura da alface, tanto em ambiente protegido como em cultivos a campo, o aumento do tamanho da parcela é uma estratégia eficiente para aumentar a precisão destes experimentos. Isso se confirma pelas estimativas das diferenças mínimas significativas entre médias de cada tamanho de parcela que, em alguns casos, reduziram seus valores em até $70 \%$ com o aumento do tamanho da parcela (Tabela 3).

Os tamanhos ótimos de parcela, estimados pelo método da máxima curvatura modificado, variaram de quatro a nove plantas dentre todos os experimentos, apresentando como valor médio de tamanho de parcela seis plantas (Tabela
3). O maior tamanho de parcela ótimo obtido neste estudo (nove plantas) é similar aos maiores tamanhos obtidos por Lúcio et al. (2004) para pimentão (dez plantas) e Mello et al. (2004) para abobrinha italiana (oito plantas).

A utilização dos tamanhos ótimos de parcela, considerando o número de repetições testado em cada experimento, em $85 \%$ dos casos resultou em diferenças mínimas significativas entre médias (D) superiores a $20 \%$, em $60 \%$ dos casos resultou em D superiores a $30 \%$ e em $45 \%$ dos casos resultou em D superiores a $40 \%$ (Tabela 3 ). Muitas pesquisas poderiam ser inviabilizadas com um D desta magnitude, visto que muitas vezes as diferenças esperadas entre médias de tratamentos são menores. Para obtenção de menores $\mathrm{D}$, as alternativas cabíveis seriam o aumento do tamanho da parcela ou do número de repetições. No caso dos ambientes protegidos, como o número de repetições de maneira geral é limitado pelo número de linhas de cultivo, que são dispostas paralelamente às aberturas laterais, resta somente a possibilidade de ajustar o número de tratamentos de modo que o tamanho de parcela possa ser aumentado.

Em cada um dos experimentos realizados e variáveis analisadas, os tamanhos de parcela possibilitaram D distintos (Tabela 3). Diante disso, para obter uma determinada precisão, são necessários tamanhos de parcela diferentes em cada variável, estação sazonal e ambiente considerado. Trabalhando com abobrinha italiana, Mello et al., (2004) também verificaram que a mudança da estação de cultivo deve ser acompanhada da mudança do tamanho 
da parcela, para que uma determinada precisão seja obtida.

$\mathrm{Na}$ avaliação dos resultados deste trabalho, verifica-se que o tamanho de parcela para alface varia em função da variável estudada, do ambiente de cultivo e da estação sazonal. Com o objetivo de que o tamanho da parcela não seja subestimado em nenhum caso, desconsiderando os D que seu uso possibilitaria, pode-se indicar o maior tamanho ótimo de parcela dentre os obtidos. Desta forma, é de oito plantas para experimentos em estufa e em túneis, e de nove plantas para experimentos a campo, o tamanho ótimo de parcela para alface, independente da variável ser Fitomassa Fresca ou Fitomassa Seca, ou da estação sazonal de cultivo.

\section{AGRADECIMENTOS}

Ao CNPq e FAPERGS, pela concessão de recursos financeiros e bolsas de iniciação científica e de produtividade em pesquisa.

\section{REFERÊNCIAS}

CARPES RH; LÚCIO AD; STORCK L; LOPES SJ; ZANARDO B; PALUDO AL. 2008. Ausência de frutos colhidos e suas interferências na variabilidade da fitomassa de frutos de abobrinha italiana cultivada em diferentes sistemas de irrigação. Revista Ceres 55: 590-595.

CARPES RH; LÚCIO AD; LOPES SJ; BENZ V; HAESBAERT FM; SANTOS D. 2010. Variabilidade produtiva e agrupamentos de colheitas de abobrinha italiana cultivada em ambiente protegido. Ciência Rural 40: 294-301.

FILGUEIRA FAR. 2000. Novo Manual de Olericultura: Agrotecnologia moderna na produção e comercialização de hortaliças. Viçosa: UFV. 402 p.

HATHEWAY WH. 1961. Convenient plot size. Agronomy Journal 53: 279-280.

LESSMAN KJ; ATKINS RE. 1963. Optimum plot size and relative efficiency of lattice designs for grain sorghum yield tests. Crop Science 3: 477-481.

LIN CS; BINNS MR. 1986. Relative efficiency of two randomized block designs having different plot size and numbers of replications and of plots per block. Agronomy Journal 78: 531-534.

LIN CS; MORRISON MJ; BINNS MR. 1996. Persistence of a field heterogeneity index. Canadian Journal of Plant Science 76: 245250.

LOPES SJ; STORCK L; HELDWEINAB; FEIJÓ S; DA ROS CA. 1998. Técnicas experimentais para tomateiro tipo salada sob estufas plásticas. Ciência Rural 28: 193-197.

LORENTZ LH; LÚCIO AD; BOLIGON AA; LOPES SJ; STORCK L. 2005. Variabilidade da produção de frutos de pimentão em estufa plástica. Ciência Rural 35: 316-323.

LÚCIO AD; CARPES RH; STORCK L; LOPES SJ; LORENTZ LH; PALUDO AL. 2008. Variância e média da massa de frutos de abobrinha-italiana em múltiplas colheitas. Horticultura Brasileira 26: 335-341.

LÚCIO AD; MELLO RM; STORCK L; CARPES RH; BOLIGON AA; ZANARDO B. 2004. Estimativa de parâmetros para o planejamento de experimentos com a cultura do pimentão em área restrita. Horticultura Brasileira 22: 766-770.

MARTIN TN; DUTRA LMC; JAUER A; STORCK L. 2005. Tamanho ótimo de parcela e número de repetições em soja (Glycine max). Ciência Rural 35: 271-276.

MEIER VD; LESSMAN KJ. 1971. Estimation of optimum field plot shape and size testing yield in Crambe abyssinica hordnt. Crop Science
11: 648-650.

MELLO RM; LÚCIO AD; STORCK L; LORENTZ LH; CARPES RH; BOLIGON AA 2004. Size and form of plots for the culture of the italian pumpkin in plastic greenhouse. Scientia Agrícola 61: 457-461.

OLIVEIRAPH; ESTEFANEL V. 1995. Tamanho e forma da parcela para avaliação do rendimento em experimentos com batata. Ciência Rural 25: 205-220.

OLIVEIRA SJR; STORCK L; LÚCIO AD; LOPES SJ; MARTINI LFD. 2006. Índice de heterogeneidade, coeficiente de variação e tamanho ótimo de parcela em batata. Ciência Rural 36: 1710-1716.

OHSE S; DOURADO-NETO D; MANFRON PA; SANTOS OS. 2001. Qualidade de cultivares de alface produzidos em hidroponia. Scientia Agricola 58: 181-185.

RESENDE MDV; SOUZA JÚNIOR CL. 1997. Número de repetições e tamanho da parcela para seleção de progênies de milho em solos sob cerrado e fértil. Pesquisa Agropecuária Brasileira 32: 1457-1461.

SANTOS D; HAESBAERT FM; PUHL OJ; SANTOS JRA; LÚCIO AD. 2010. Suficiência amostral para alface cultivada em diferentes ambientes. Ciência Rural 40: 800-805.

SMITH HF. 1938. An empirical law describing heterogeneity in the yields of agricultural crops. Journal of Agricultural Science 28: $1-23$.

STEEL RGD; TORRIE JH; DICKEY DA. 1997. Principles and procedures of statistics: a biometrical approach. 3.ed., Nova York: McGraw-Hill. 666p.

STORCK L; GARCIA DC; LOPES SJ; ESTEFANEL V. 2006. Experimentação vegetal. 2.ed. Santa Maria: Ed. da UFSM. 198p.

ZANON MLB; STORCK L. 2000. Tamanho ótimo de parcelas experimentais para Eucalyptus saligna Smith em dois estádios de desenvolvimento. Cerne 6: 104-111.

ZHANG R; WARRICK AW; MYERS DE. 1994. Heterogeneity, plot shape effect and optimum plot size. Geoderma 62: 183-197. 